\title{
The Role of Augmented Feedback on Motor Skill Learning
}

\author{
Yadi Sunaryadi \\ Coaching Department, Faculty of Physical Education and Sport \\ Universitas Pendidikan Indonesi \\ Bandung, Indonesia \\ ydsunaryadi@upi.edu
}

\begin{abstract}
The purpose of this literature study was to examine whether augmented feedback enhances motor skill learning in sports, and if so, how this can be explained. When athletes or students perform a skill, they receive two types of performance-related feedback. One type of feedback is called task-intrinsic feedback, which is the sensory information that is a natural part of performing a skill (e.g. vision, audition, and proprioception). The second type of performance-related feedback is called augmented feedback. Augmented feedback is a means of supplementing the sources of task-intrinsic feedback normally available to the learner. The conclusion suggests that augmented feedback is necessary for learning some skills. In optimizing the way in which augmented feedback is presented to the learner, therefore, the selection of the type of feedback carefully must be emphasized
\end{abstract}

Keywords - Augmented feedback; knowledge of Result (KR); Knowledge of Performance (KP); motor skill learning

\section{INTRODUCTION}

One of the most important variables in the motor-learning process is the feedback provided to the learner attempting to acquire a new motor skill [1]. Feedback refers to any information received by the learner before, during, and after an attempt to perform a task is information about the quality or quantity of the performance which generally believed to be one of the most important factors in guiding the process of learning motor skills [2]. Once a movement skill is performed it is appropriate for the physical educator or coach to provide feedback to the students or athletes. Feedback refines the motor system by guiding the learner towards a movement pattern in a manner that promotes implicit (self-correcting) processes [3]. It is classically broken down into two categories comprised of task-intrinsic feedback and augmented feedback (external or artificial feedback). Intrinsic feedback is considered information that obvious to the learner and is received across visual, auditory, tactile, and proprioceptive sensory inputs [4]. It is unquestionable that provides feedback on information which is apparent to the learner can be considered redundant and therefore can be avoided. As we know that student's or athlete's working memory and attention are limited in capacity and for this reason it is critical that instructors must limit information to what is necessary for learner's success. Consequently, this variable has received a great amount of interest in the research literature [5].

It is important to know that the expression 'practice makes perfect' is not commonly used anymore in sport related context. Only the expression 'practice doesn't make perfect, perfect practice makes perfect' is the most commonly used in a variety of sport related context. This expression implies that properly structured practice facilitates motor skill learning, which ultimately culminates in enhanced motor skill performance [6]. Most sports scientists and coaches agree that the conditions of practice influence motor skill acquisition; because of this, practitioners must take many factors into consideration when creating practice schedules to develop an athlete's performance and prepare him or her for competition [7]. Coaches have two options when providing external feedback to their athletes. They can either provide knowledge of performance (KP) or knowledge of results (KR) feedback. Knowledge of performance (KP) is information an athlete receives about their performance that is specifically related to their movements while knowledge of results (KR) is information the athlete receives about the performance outcome. One method of providing KP to athletes is providing them information about the kinematics of their movements. This may include body or limb position, velocity, or acceleration [8]. While KP provides information about movement characteristics of an athlete's performance, KR provides coaches a means of conveying outcome related information to athletes. Examples of outcome related information may include but not limited to: how fast a sprinter ran an event, distance an implement was thrown, how fast a tennis serve executed by a tennis player [9]. As physical education teachers or coaches, we are all aware that one of our most important roles in the process of motor skill learning is to give the learners or athletes with feedback information about their learning performance. 


\section{PRESS}

It is common to provide people laming skills with some type of feedback about their skill performances. When used in this way, feedback can be referred to as augmented feedback, although a variety of other terms have been used to refer to the same type of externally presented feedback [14]. It is called augmented feedback because the information provided by the physical educator or coach "augments" the sensory feedback the student or athlete obtains naturally [10]. For the purpose of this paper augmented feedback will be referred to as "feedback". In fact, augmented feedback has traditionally been given a two-part role in skill learning. One part is that augmented feedback is necessary for effective skill learning to occur. The second is that augmented feedback is beneficial for skill learning. The performance of a skill is influenced, and often determined by the information provided as feedback from external source, such as a physical education teacher or coach. Although there are two types of information in augmented feedback, it is KR feedback that has been the primary focus for the extensive research in the area of motor skill learning in the past due to the lack of separation of KR and KP information operationally and experimentally. Therefore, it is argued that what we understand at present about augmented feedback is actually the effect of KR information in situations of learning simple "artificial" tasks in a laboratory setting.

There is an obvious need to examine the effects of feedback types on real-life skill in further research of motor learning. The fact that KP information is frequently used by physical education teachers and coaches in practical settings suggests that KP feedback is seen to provide more useful information than KR feedback for learning motor skills. [11] Unfortunately, studies of KP feedback actually did not yield consistent positive results with respect to the function of several types of KP information such as videotape replay, verbal KP, kinematic and kinetic KP in motor learning. [12] Therefore, how $\mathrm{KP}$ feedback is used by learners or how KP enhances motor skill learning is not well understood at the present time and many questions are in need of being addressed by more research. The most prominent criticisms of past research on augmented feedback, along with the lack of separation of KR and KP, and the lack of retention or transfer tests, are about the use of "artificial" laboratory tasks and the lack of consideration of the characteristics of motor skills. It has been argued that the generalized principles of augmented feedback from the laboratory with one-dimensional tasks may not apply well to more complex real-world settings [9-25], and that skills with different characteristics need different types of feedback information for effective learning. The number of explanation for the lack of effectiveness on skill acquisition may be due to overly complex information presented, failure to provide critical feedback about the skill and not providing enough information for error detection [13]. Thus, the importance of carefully selecting the type of feedback must be emphasized. In this section, we will review findings related to the the influence of the augmented feedback induced by instructions or feedback on motor skill learning.

\section{AUGMENTED FEEDBACK REVIEW}

As we know that "learning" as the ability to detect and correct failures. One of augmented feedback, the Knowledge of Results (KR) was considered by many authors in 60's and 70's [14] and, more recently, in 80's [15], was deemed as one of the most important variables to control and acquire motor skills. Given the concerns acquired by the influence of KR on learning processes, it was even asserted that the more frequency, accuracy and immediacy when providing KR, the better learning rates would be reached. Several studies qualified this direct and positive relation of KR with the learning of motor skills. Firstly, different researches showed that motor learning could occur without KR [29]. In fact, this kind of studies, instead of the lack of KR, demanded the importance of the inherent information obtained by the subject from his own movement when acquiring a skill (internal feedback or IFB). However, it has been proved that, in predominantly inherent time anticipation tasks, the subjects with $0 \%$ of KR learned at a lesser extent than those with $100 \%$ of KR. Some studies even showed that providing KR during the acquisition of a skill (external feedback or EFB) had more influence than the subject's inherent information itself, although this could be enough to learn the task and the provided KR could be incorrect or redundant with the IFB. Secondly, the inclusion of retention tests when analyzing the tasks showed that a precise KR, large and immediate, contributed to a positive effect on the acquisition, although was a negative influence on the retention of the tasks to be learned. This is called the "double effect" of KR, which is not valid for every kind of tasks. Kohl and Guadagnoli [16] carried out a research with three experimental groups provided with $100 \%$ of KR, $50 \%$ (6 repetitions with KR and 6 without), and 50\% (12 with KR and 12 without KR), respectively. The analysis of the 6 and 12 first attempts showed that the third group was better than the first one, which was for its part better than the second group; moreover, the retention of the first group (100\% of KR) was better than the retention of the second group (50\% of KR). Also Badets, Blandin, Wright, and Shea [17] as shown in the retention phase after learning, subjects who received half the KR show greater stability in the results.

Given these aspects, Magill pointed out that KR can be essential when acquiring skills if the information provided by the IFB is not enough or cannot be used by the subject. It may not be required to acquire skills when the IFB is enough, evident and achievable, becoming the EFB a redundant information without usefulness. At the same time, it can help the acquisition of skills, without resulting essential or redundant, while accelerating the learning processes of basically inherent skills; it may also interfere the skill acquisition by creating a dependence of the $\mathrm{EFB}$, which causes a reduction of performance. These 
changeable results when applying KR seem to depend on the complexity of the task. According to Swinnen [18], the complexity of the task is related to the richness of the provided IFB - the more complexity of the task, the more richness of the IFB; he also asserted that the tasks with a simple IFB (less complex tasks) could be more susceptible to the negative effects of KR than the tasks with a complex IFB. To go into this aspect in more depth, we analyzed the different variables of application of KR on simple and complex tasks. Wulf, Shea, and Matschiner [19] studied the influence of the KR frequency on learning the complex skill of skiing slalom; they found out that the group with $100 \%$ of KR had a better performance than the group with $50 \%$, and this one performed better than the group with $0 \%$ during retention tests without KR. We could reach the conclusion that a high frequency of KR improves the acquisition while reduces the retention in simple tasks, although when these tasks are complex and less consolidated, a high frequency of KR would lead to improvements during the acquisition and retention stages [20]. One of the variables of the application of KR more often analyzed is the accuracy of the information provided to the trainee. As well as for the assessment of the frequency variable, there are several studies asserting that it cannot be established a direct relation between the accuracy level and learning [21]. The studies by Rogers (1974) raised the theory of an ideal accuracy level of KR, above and below which the learning rate is reduced. However, some replicas made of these studies argued that, if the subjects know the meaning of the units used to provide KR, there would be no differences in learning between the groups with a higher or lower accuracy level of KR. Reeve and Magill carried out a study about the influence of providing information related to the distance or direction of the failure events in a simple positioning test of a blind implement. These authors' conclusion established that KR about the direction of the failure events (less precise) is more useful in the initial stage of the learning process, while KR about the distance of the failure events (more precise) requires a certain level of experience in the subjects, so they can acquire it as useful information. By providing significant KR to the trainee and carrying out retention tests, Magill and Wood obtained similar results in a study about the influence of providing quantitative or qualitative KR in a complex task of reproduction of a specific movement. There were no main differences between the groups during the acquisition stage, while in the retention stage; the group with quantitative KR was better than the group with qualitative one. These results confirmed that both kinds of KR would be useful at the same degree, since they provided information about the direction of the failure events (less precise) at the initial learning stages; moreover, at the end of the acquisition stage and during retention, the quantitative information would be more useful than qualitative, since it provided information about the magnitude of the failure events (more precise). Reeve, Dornier, and Weeks [22] came to a similar conclusion asserting that the precision of $\mathrm{KR}$ influenced on learning in a way that, at the earlier learning stages, a high accuracy level could be negative but, from a significant and durable point of view, the more accuracy provided, the more learning would be reached. Moreover, Wulf [23] pointed out the importance of providing more information about success events than about failures. Wright et al., concluded that quantitative KR was useful when the failure was high, but that it was impossible to assert that qualitative KR would be more useful at long-term than quantitative when correcting small failure events.

The role of augmented feedback to facilitate skill acquisition and learning is a widely accepted axiom. Many professionals from the areas of motor learning and pedagogy can investigate to the strength of both KP and KR to promote skill performance and learning. In fact, one key role a sport instructor or physical educator can play is that of delivering augmented KP feedback regarding the characteristics of a movement pattern to a student attempting to acquire a motor skill [24]. Unfortunately, the degree of accuracy, frequency, and type of KP feedback delivered by many physical educators is less than desirable. The findings of a descriptive study on augmented feedback conducted by Fishman and Tobey revealed that instructors delivered feedback (all types of feedback- KP, KR, and behavioral) at a rate of 1.5 per minute. In addition, Fishman and Tobey reported that over half $(53 \%)$ of all feedback delivered to students was evaluative in nature and addressed performance outcome (KR) (e.g., nice throw, good shot, you scored 19 points), leaving a smaller percentage (42\%) of feedback dedicated to information on the execution of a movement pattern (KP). Several studies have investigated the effect of kinematic information feedback upon skill acquisition during practice. Primarily, these researchers utilized KP to inform subjects about performance errors in movement pattern(s) while subjects practiced a motor task in a laboratory setting. The findings of all but one of these investigations revealed that, during practice, KP promoted skill performance as compared to a no-KP feedback control condition. In a recent study, Young and Schmidt utilized a coincidental-timing task with three schedules of KP feedback. The findings indicated no significant differences among the three KP schedules during the practice phase of the experiment. In addition, these researchers reported that, although no significant fmdings existed among the three feedback schedules, subjects receiving KP after every trial exhibited lower scores as compared to subjects in the other two feedback conditions (average score after five trials and average fade). However, during retention, students receiving KP after every trial scored significantly lower than those receiving the other two schedules of feedback. Although the research on KR has been prolific, the research on KP has been paltry [25]. The lack of research on KP may be partly due to the redefinition of KR, which encompassed some types of augmented KP. Schedules of feedback have been the topic of many KR investigations. Briefly, there are two opposing theories regarding the impact of feedback schedules upon performance and learning. Thorndike proposed that 
feedback should be delivered after every trial because it would enhance learning by strengthening the memory trace. Further, he contended that as the frequency of feedback increased so would the amount of learning. The early research of Bilodeau and Bilodeau compared the performance scores of subjects that received KR after every trial, every 3rd and 4th trial, or every 10th trial. Their research findings indicated that performance only improved when each trial was immediately followed by KR. In contrast, the findings of other research [26] have not supported the employment of KR after every trial because the use of KR may cause dependence and actually detract from learning by limiting the subject's utilization of internal sensory feedback for error detection and correction. Some evidence exists to support the postulate that augmented KP plays a crucial role in the acquisition of a closed motor skill [27]. In addition, Schmidt, addressing the research potential of KP and KR, stated that "when extensive practice at some sport or athletic activity, it would seem far more effective to provide information about ... $\mathrm{KP}$. Why the focus on KR . . when KP . . . is what will probably be the most useful for application"

\section{IMPLICATIONS FOR SKILL INSTRUCTION}

With regard to the quality of practice, a potential way to support the motor learning process is to provide a student or athlete with augmented feedback that supplements the response - produced inherent feedback obtained from vision, audition, and proprioception [28]. The learner can achieve a certain skill of level with task- intrinsic feedback, but in order to attain a higher level of expertise, augmented feedback is needed. In an instructional situation, it is important to determine what augmented feedback to provide and when to provide it. The previous discussion of the various relationships between augmented feedback and skill learning provides some direction for making these critical determinations. Three suggestions according to Magill are evaluate the skill, evaluate augmented feedback characteristic, and evaluate the meaningfulness of augmented feedback. Furthermore, he explained that if the skill being learned is difficult for the learner to detect critical sensory feedback information, such as when a limb's spatial position cannot be seen, then augmented feedback is required; if the skill being learned involves acquiring a new concept that is essential for successful performance, such as understanding a unit of measurement, then again, augmented feedback is required; if the skill provides the learner with all the essential feedback information needed to learn the skill, then augmented feedback may not be needed. Without this type of augmented feedback, the skill can be learned to a limited degree; however, the availability of augmented feedback based on limb movement characteristics enhances the level of performance achieved. In these situations, what becomes critical to facilitate learning is determining what information to give as augmented feedback and how to give it. Evaluating the augmented feedback that will be provided in a situation to determine if the feedback may attract the learner's attention is very important. It would be a good situation if the instructor know how different forms of augmented feedback influence learning a particular skill. A qualified physical education teacher or coach typically use a variety of means to provide augmented feedback. Some are in the form of verbal, and others are visual. Preparing effective augmented feedback clearly requires physical education teacher or coach's knowledge of both the skill and augmented feedback. The presence of augmented feedback attracted attention to such an extent that incorrect information was not evaluated as incorrect but was used as the basis for performing the skill. What this means is that instructors must provide suitable information when giving feedback. Augmented feedback is a form of informatin to the learner about his or her performance of the skill being learned. Thus, it is arising as a consequence of performance. It is therefore important to consider what kind of information should be given and the most influence on the learner. Instructors seem to have this characteristic as they gain experience and become more aware of how to determine instructional strategies that best facilitate skill learning. The stage of an individual's learning is also an important learner characteristic for determining what augmented feedback is meaningful. Whether the learner is at cognitive stage, associative stage, or autonomous stage. The information given to subjects prescribed what needed to be done to correct an important performance error. Thus, the most suitable feedback for beginners is prescriptive information that is important to facilitate learning. The feedback should contain information that will allow the learner to determine what needs to be done on the next trial to improve performance. This type of information is distinct from what is known as descriptive knowledge of performance, which simply describes the error that must be corrected. It describes the error that needs correcting but does not give any information about what to do to make the correction [29]. For the beginner, this type of information is of little value, but it can be very useful for the skilled person. Although descriptive KP directs a person's attention to the error that needs correcting, only the skilled person has the knowledge needed to make the appropriate correction.

\section{CONCLUSION}

In conclusion, augmented feedback is often provided to boost performance during skill acquisition. Providing augmented feedback during training improves performance, whereas its removal during a subsequent test or retention condition may result in performance deterioration.

The most important feature of information that must be given to the students or athletes is the appropriateness of augmented feedback. When augmented feedback is needed, the critical concern must be to determine three things: what information to give, how to give it, and how often to give it. The answers to 


\section{B ATLANTIS PRESS}

these important questions should be based on knowledge of the skill being learned, the effects different types of augmented feedback will have on learning that skill, and the characteristics of the individuals learning the skill. The information must be appropriate for the skill being learned, the person learning the skill, and the learning situation. These information will help physical education teachers or coaches to meet the specific needs and demands of students or athletes during motor learning practice, which in turn, enlarge enhanced performance during learning or competition.

\section{ACKNOWLEDGMENT}

The article reported was made as part of the first author's doctoral dissertation at Universitas Pendidikan Indonesia. Thanks are extended to Beltasar Tarigan, Yudy Hendrayana, Gabriele Wulf, and an anonymous reviewer for their many helpful suggestions on an earlier draft of this manuscript.

\section{REFERENCES}

[1] Bilodeau E A, Bilodeau I M, and Schumsky D A 1959 Some effects of introducing and withdrawing knowledge of results early and late in practice. Journal of Experimental Psychology, 58, 142-144.

[2] Reeve T G, Domier L A and Weeks D J 1990 Precision of knowledge of results:Consideration of the accuracy requirements imposed by the task. Research Quarterly for Exercise and Sport

[3] Magill R A 2012 The influence of augmented feedback on skill learning depends on characteristics of the skill and the learner. National Association for Physical Education in Higher Education. Quest, 46, 323326

[4] Magill R A 2012 The influence of augmented feedback on skill learning depends on characteristics of the skill and the learner. National Association for Physical Education in Higher Education. Quest, 46, 323326

[5] Swinnen S 1996 Information feedback for motor skill learning: a review in H.N Zelaznik (Ed). Advances in motor learning \& Control. Champaign: Human Kinetics

[6] Wright D L, Smith-Munyon, V.L and Sidaway B 1997 How Close is Too Close for Precise Knowledge of Results?. Research Quarterly for Exercise and Sport. Volume 68, Issue 2.

[7] Ho and Shea 1978 Effects of relative frequency of KR on retention of a motor skill. Perceptual Motor Skills

[8] Schmidt R A, and Young E D 1990 Augmented kinematic feedback for motor learning. Journal of Motor Behavior, 24:261-273

[9] Reeve T G, Domier L A and Weeks D J 1990 Precision of knowledge of results:Consideration of the accuracy requirements imposed by the task. Research Quarterly for Exercise and Sport
[10] Newell K M and Walter C B 1981 Kinematic and kinetic parameters as information feedback in motor skill acquisition. Journal of Human Movement Studies, 7, 235-254.

[11] Fishman S and Tobey C 1978 Augmented feedback. In W. G. Anderson \& G. Barrette (Eds.), What's going on in gym: Descriptive studies of physical education classes [Monograph]. Motor Skills: Theory Into Practice, 1, 51-62

[12] Schmidt R A 1988 Motor Control \& Learning: A behavioral emphasis (2nd ed). Champaign. I.L. Human Kinetics

[13] Reeve T G, Domier L A and Weeks D J 1990 Precision of knowledge of results:Consideration of the accuracy requirements imposed by the task. Research Quarterly for Exercise and Sport

[14] Bilodeau I M 1969 Information feedback. In E.A Bilodeau (Ed). Principle of skill acquisition. New York: Academic Press

[15] Rucci J A and Tomporowski, P.D 2009 Effects of three different types of kinematic feedback on the execution of the hang power clean. Unpublished master's thesis.

[16] Guadagnoli M, Leis B, Van Gemmert A W A, and Stelmach G E 2010 The relationship between knowledge of results and motor learning in Parkinsonian patients. Parkinsonism and Related Disorders, 9, 89-95.

[17] Badets A, Blandin Y, Wright D L, and Shea C H 2006 Error detection processes during observational learning. Research quarterly for exercise and sport, 177-184

[18] Schmidt R A and Young E D 2010 Methodology for motor learning: A paradigm for kinematic feedback. Journal of Motor Behaviour, 23, 1324.

[19] Wulf G ,Schmidt R A and Deubel H 1993 Reduced feedback frequency enhances generalized motor program learning but not parameterization learning. Journal of Experimental Psychology: Learning, memory, and cognition, 19.

[20] Anderson J R et al 2001 Skill acquisition: Compilation of weak-method problem solutions. Psychological Review 94, 192-210.

[21] Wright D L, Snowden S and Willoughby D 1990 Summary KR: how much information is used from the summary ? Journal of Human Movement

[22] Porter J M, Wu, W E W, and Partridge J A 2010 Focus of attention and Verbal instructions: Strategies of elite track and field coaches and athletes. Sport Science Review, Vol XIX, No.3-4

[23] Wulf G, Shea, C.H, Matschiner S 1998 Frequent feedback enhances complex motor skill learning. Journal of Motor Behavior, 30, 180-192

[24] Boyce B A 1987 Effect of two instructional strategies on acquisition of a shooting task. Perceptual and Motor Skills, 65

[25] Salmoni A, Schmidt R A, and Walter CB 1984 Knowledge of results and motor learning: A review and reappraisal. Psychological Bulletin, 95, 355-386.

[26] Hatze H 1976 Biomechanical aspects of a successful motion optimization. In:P.V. Komi (ed.), Park Press.

[27] Thorndike 1927 The law of effect. American Journal of Psychology, 39

[28] Mononen K 2007 The effects of augmented feedback on motor skill learning in shooting. A feedback training intervention among inexperienced rifle shooters. University Jyvaskyla

[29] Wrisberg C 2010 Sport Skill Instruction for Coaches, Human Kinetics 